\title{
Design of IIR multiband filters using IIR all-pass filters
}

\author{
Soo-Chang Pei*, a , Jong-Jy Shyu ${ }^{\mathrm{b}}$ \\ ${ }^{a}$ Department of Electrical Engineering (Room 319), National Taiwan University, Taipei, Taiwan, ROC \\ ${ }^{b}$ Department of Computer Science and Engineering, Tatung Institute of Technology, Taipei, Taiwan, ROC
}

Received 2 November 1992; revised 25 March 1993 and 8 July 1993

\begin{abstract}
An efficient method is proposed to design IIR all-pass filters for synthesizing general multiband filters which are of low sensitivity. The method is based on minimizing a quadratic measure of the error derived from the phase design of certain filter. The problem of stability is of no concern, and the performance of the obtained filter is comparable to that of the existing methods. Moreover, the solution is obtained directly with no need for iteration.
\end{abstract}

\section{Zusammenfassung}

Es wird eine effiziente Methode zum Entwurf rekursiver Allpässe für die Synthese allgemeiner Mehrbandfilter mit geringer Empfindlichkeit vorgeschlagen. Sie basiert auf der Minimierung eines quadratischen Fehlermaßes, das aus dem Phasenentwurf gewisser Filter abgeleitet wird. Das Problem der Stabilität spielt keine Rolle und die Qualität der gewonnenen Filter ist mit bekannten vergleichbar. Darüber hinaus wird die Lösung direkt, also ohne Iteration gewonnen.

\section{Résumé}

Nous proposons une méthode efficiente de conception de filtres passe-tout IIR pour la synthèse de filtres multi-bandes généraux ayant une faible sensibilité. Cette méthode est basée sur la minimisation d'une mesure quadratique de l'erreur dérivée de la conception en phase d'un certain filtre. Le problème de la stabilité n'entre pas en ligne de compte, et les performances du filtre obtenu sont comparables à celles des méthodes existantes. De plus, la solution est obtenue directement sans aucune itération.

Key words: IIR multiband filter; IIR all-pass filter; Eigenfilter approach; Halfband filter; Complex IIR multiband filter

\section{Introduction}

There is an efficient structure for implementing IIR multiband filters by two all-pass sections [1-4,

*Corresponding author.
10, 11]. The advantages of this structure are low sensitivity and less computation. In this paper, we will use the eigenfilter approach to design the two all-pass sections. The eigenfilter approach has been successfully used to design the linear-phase filters, Hilbert transformers and differentiators $[6,7,9]$. Instead of minimizing a quadratic measure of the 
error of amplitude response over the passband and stopband $[6,7,9]$, the proposed eigenfilter approach is based on minimizing a quadratic measure of the error based on the phase design of certain filter. Because the problem of stability is of no concern, the method is easy and efficient, and the overall performance is comparable to the existing methods. Moreover, one of the main advantages of the proposed method is that the solution is obtained directly without the need for iteration. Also the method can be extended easily to design complex multiband filters by two parallel complex IIR all-pass sections.

This paper is organized as follows. Section 2 describes the basic structure for implementing multiband filters by two all-pass sections, and the constraints for the filter orders. It is shown that the design problem can be solved by approaching a phase response. The design algorithm is extended to design complex multi-band filters by using two complex all-pass sections in Section 3. Several examples are presented to demonstrate the usefulness of the eigenfilter approach. Finally, the conclusions are given in Section 4.

\section{Problem formulation to the design of multiband filters using IIR all-pass filters}

The proposed filter is based on a parallel interconnection of two all-pass filters and the transfer function is denoted by

$H(z)=\frac{1}{2}\left[H_{1}(z)+(-1)^{I} H_{2}(z)\right], \quad I=0,1$,

where $H_{1}(z)$ and $H_{2}(z)$ are stable all-pass sections with the transfer functions [8] as follows:

$H_{1}(z)=z^{-M} \frac{\sum_{n=0}^{N_{1}} a_{1}\left(N_{1}-n\right) z^{-n}}{\sum_{n=0}^{N_{1}} a_{1}(n) z^{-n}}$

and

$H_{2}(z)=\frac{\sum_{n=0}^{N_{2}} a_{2}\left(N_{2}-n\right) z^{-n}}{\sum_{n=0}^{N_{2}} a_{2}(n) z^{-n}}$,

in which $M$ is usually either 0 or 1 . Eq. (1) can be rewritten as

$H(z)=\frac{1}{2} H_{1}(z)\left[1+(-1)^{\prime} \frac{H_{2}(z)}{H_{1}(z)}\right]$

$$
\begin{aligned}
& =\frac{1}{2} H_{1}(z)\left[1+(-1)^{I} G(z)\right] \\
& =\frac{1}{2} H_{1}(z) U(z),
\end{aligned}
$$

where $G(z)$ is also obviously in all-pass filter and given by

$G(z)=\frac{H_{2}(z)}{H_{1}(z)}=z^{M} \frac{\sum_{n=0}^{N} a(N-n) z^{-n}}{\sum_{n=0}^{N} a(n) z^{-n}}$,

in which $N=N_{1}+N_{2}$ and

$a(n)=a_{1}\left(N_{1}-n\right) * a_{2}(n)$.

* denotes the convolution operation. It is noted that although $H_{1}(z)$ and $H_{2}(z)$ are both assumed stable, $G(z)$ is unstable.

To design multiband filters, certain constraints for the choice of $I, M$ and filter order should be considered. Due to the all-pass characteristic of $H_{1}(z)$ in (4), the overall amplitude response of $H(z)$ is determined by $U(z)$. Notice that

$U\left(\mathrm{e}^{\mathrm{j} 0}\right)=U(+1)=1+(-1)^{I}$

and

$U\left(\mathrm{e}^{\mathrm{j} \pi}\right)=U(-1)=1+(-1)^{I+M+N}$,

hence for different filter responses the following constraints should be satisfied:

$$
\begin{aligned}
\left|H\left(\mathrm{e}^{\mathrm{j} 0}\right)\right| & =1 \text { and }\left|H\left(\mathrm{e}^{\mathrm{j} \pi}\right)\right|=0: \\
U(1) & =2, U(-1)=0, \\
\left|H\left(\mathrm{e}^{\mathrm{j} 0}\right)\right| & =0 \text { and }\left|H\left(\mathrm{e}^{\mathrm{j} \pi}\right)\right|=1: \\
U(1) & =0, U(-1)=2, \\
\left|H\left(\mathrm{e}^{\mathrm{j} 0}\right)\right| & =\left|H\left(\mathrm{e}^{\mathrm{j} \pi}\right)\right|=0: \quad U(1)=0, U(-1)=0, \\
\left|H\left(\mathrm{e}^{\mathrm{j} 0}\right)\right| & =\left|H\left(\mathrm{e}^{\mathrm{j} \pi}\right)\right|=1: \quad U(1)=2, U(-1)=2,
\end{aligned}
$$

which lead to a set of constraints on $I, M$ and $N$ as summarized in Table 1. For a low-pass filter design, for example, $I=0$ and $M+N$ must be odd.

We now take the design. Because, the amplitude response of $H(z)$ is determined by $U(z)$, it is appropriate to design $G(z)$ rather than to design $H_{1}(z)$ and $H_{2}(z)$ separately. Also, due to the fact that $G(z)$ is also an all-pass filter, the design problem reduces to design the phase of $G(z)$ only. For the passband design, 1 and $(-1)^{I} G(z)$ must be in-phase. On the other hand, 1 and $(-1)^{I} G(z)$ must be out-phase for 
Table 1

Constraints on $I, M$ and $N$ for different filter response

\begin{tabular}{lll}
\hline Filter response & $I$ & $M+N$ \\
\hline$\left|H\left(\mathrm{e}^{\mathrm{j} 0}\right)\right|=1$ and $\left|H\left(\mathrm{e}^{\mathrm{j} \pi}\right)\right|=0$ & 0 & Odd \\
$\left|H\left(\mathrm{e}^{\mathrm{j} 0}\right)\right|=0$ and $\left|H\left(\mathrm{e}^{\mathrm{j} \pi}\right)\right|=1$ & 1 & Odd \\
$\left|H\left(\mathrm{e}^{\mathrm{j} 0}\right)\right|=\left|H\left(\mathrm{e}^{\mathrm{j} \pi}\right)\right|=0$ & 1 & Even \\
$\left|H\left(\mathrm{e}^{\mathrm{j} 0}\right)\right|=\left|H\left(\mathrm{e}^{\mathrm{j} \pi}\right)\right|=1$ & 0 & Even
\end{tabular}

the stopband design. Moreover, it is unnecessary to concern the stability of $G(z)$. Because we can split the obtained $G(z)$ into two all-pass sections, $G_{1}(z)$ and $G_{2}(z)$, where $G_{1}(z)$ has only those poles that lie outside the unit circle and $G_{2}(z)$ has poles inside the unit circle, and then set

$H_{1}(z)=\frac{1}{G_{1}(z)}$

and

$H_{2}(z)=G_{2}(z)$.

Let

$F(z)=\sum_{n=0}^{N} a(n) z^{-n}$.

Eq. (5) becomes

$G(z)=\frac{z^{M-N} F\left(z^{-1}\right)}{F(z)}$

and

$\arg \left(G\left(\mathrm{e}^{\mathrm{j} \omega}\right)\right)=(M-N) \omega-2 \arg \left(F\left(\mathrm{e}^{\mathrm{j} \omega}\right)\right)$.

So the phase design of $G(z)$ can be insteaded by the phase design of $F(z)$. Assume the desired phase for designing $G(z)$ is $P_{G}(\omega)$, then, equivalently, the desired phase for designing $F(z)$ is

$P_{F}(\omega)=\frac{(M-N) \omega-P_{G}(\omega)}{2}$

The actual phase of $F(z)$ can be represented by

$$
\begin{aligned}
\arg \left(F\left(\mathrm{e}^{\mathrm{j} \omega}\right)\right) & =\tan ^{-1} \frac{-\sum_{n=0}^{N} a(n) \sin (n \omega)}{\sum_{n=0}^{N} a(n) \cos (n \omega)} \\
& =P_{a}(\omega) .
\end{aligned}
$$

The alternative representation of (16) is

$$
\frac{-\sum_{n=0}^{N} a(n) \sin (n \omega)}{\sum_{n=0}^{N} a(n) \cos (n \omega)}=\tan \left(P_{a}(\omega)\right)=\frac{\sin \left(P_{a}(\omega)\right)}{\cos \left(P_{a}(\omega)\right)},
$$

so

$$
\begin{gathered}
\sin \left(P_{a}(\omega)\right) \sum_{n=0}^{N} a(n) \cos (n \omega)+\cos \left(P_{a}(\omega)\right) \\
\times \sum_{n=0}^{N} a(n) \sin (n \omega)=0
\end{gathered}
$$

Defining

$A=\left[\begin{array}{llll}a(0) & a(1) & \ldots & a(N)\end{array}\right]^{T}$

and

$C(\omega)=\left[\begin{array}{llll}c(0) & c(1) & \ldots & c(N)\end{array}\right]^{\mathrm{T}}$,

where

$$
\begin{aligned}
c(i) & =\sin \left(P_{a}(\omega)\right) \cos (i \omega)+\cos \left(P_{a}(\omega)\right) \sin (i \omega), \\
i & =0, \ldots, N,
\end{aligned}
$$

then Eq. (18) can be rewritten as an inner product form:

$A^{\mathrm{T}} C(\omega)=0$.

But it is impossible for finite $N$ to realize $F(z)$ such that the actual phase $P_{a}(\omega)$ of $F(z)$ is exactly equal to the desired phase $P_{F}(\omega)$ of $F(z)$, and the problem we face is to replace $P_{a}(\omega)$ in (21) by $P_{F}(\omega)$ and find a set of filter coefficients to minimize $\boldsymbol{A}^{\mathrm{T}} \hat{\boldsymbol{C}}(\omega)$ as possible, where

$\hat{C}(\omega)=\left[\begin{array}{llll}\hat{c}(0) & \hat{c}(1) & \ldots & \hat{c}(N)\end{array}\right]^{\mathrm{T}}$,

in which

$$
\begin{aligned}
\hat{c}(i) & =\sin \left(P_{F}(\omega)\right) \cos (i \omega)+\cos \left(P_{F}(\omega)\right) \sin (i \omega), \\
i & =0, \ldots, N .
\end{aligned}
$$

In this paper, we use the least-squares approach to this problem with the error function

$$
\begin{aligned}
E & =\int_{r} W(\omega)\left[\boldsymbol{A}^{\mathrm{T}} \hat{\boldsymbol{C}}(\omega)\right]^{2} \mathrm{~d} \omega \\
& =\boldsymbol{A}^{\mathrm{T}}\left[\int_{r} W(\omega) \hat{\boldsymbol{C}}(\omega) \hat{\boldsymbol{C}}^{\mathrm{T}}(\omega) \mathrm{d} \omega\right] \boldsymbol{A} \\
& =\boldsymbol{A}^{\mathrm{T}} \boldsymbol{Q} \boldsymbol{A},
\end{aligned}
$$


where $r$ represents the region of design bands, $W(\omega)$ is a weighting function and $Q$ is a real, symmetric and positive-definite matrix [5]. Hence, the eigenfilter approach $[6,7,9]$ can be applied to design the phase of $F(z)$. Notice that the elements of $Q$ are given by

$$
\begin{array}{rl}
q(n, m)=\int_{r} & W(\omega)\left[\sin \left(P_{F}(\omega)\right) \cos (n \omega)\right. \\
& \left.+\cos \left(P_{F}(\omega)\right) \sin (n \omega)\right] \\
& \times\left[\sin \left(P_{F}(\omega)\right) \cos (m \omega)\right. \\
& \left.+\cos \left(P_{F}(\omega)\right) \sin (m \omega)\right] \mathrm{d} \omega,
\end{array}
$$

$$
0 \leqslant n, m \leqslant N \text {. }
$$

It is difficult to derive the closed forms of $q(n, m)$, so that numerical analysis must be applied. In this paper, 200 samples are used between $\omega=0$ and $\pi$.

EXAMPLE 1. This example deals with the design of a low-pass filter with passband $[0,0.3 \pi]$ and stopband $[0.5 \pi, \pi]$ in normalized frequency. The parameters chosen are $I=0, M=1, N=N_{1}+N_{2}$ $=8$ according to the constraints listed in Table 1 . Obviously, the desired phase of $G(z)$ is

$P_{G}(\omega)= \begin{cases}0, & \omega \in \text { passband } \\ \pi, & \omega \in \text { stopband }\end{cases}$

Due to the increasing deviation tending to the band edges for the least-squares approach, the weighting function is properly chosen as

$W(\omega)= \begin{cases}\omega, & \omega \in \text { passband } \\ \pi-\omega, & \omega \in \text { stopband }\end{cases}$

By computer simulation, we found that the obtained result is insensitive to another weighting function we chose. For the transition band $[0.3 \pi, 0.5 \pi]$, it is just taken as a 'don't care' band. The obtained poles of $G(z)$ outside the unit circle are

$0.0470014 \pm \mathrm{j} 1.008618$

$1.334092 \pm \mathrm{j} 1.748175$
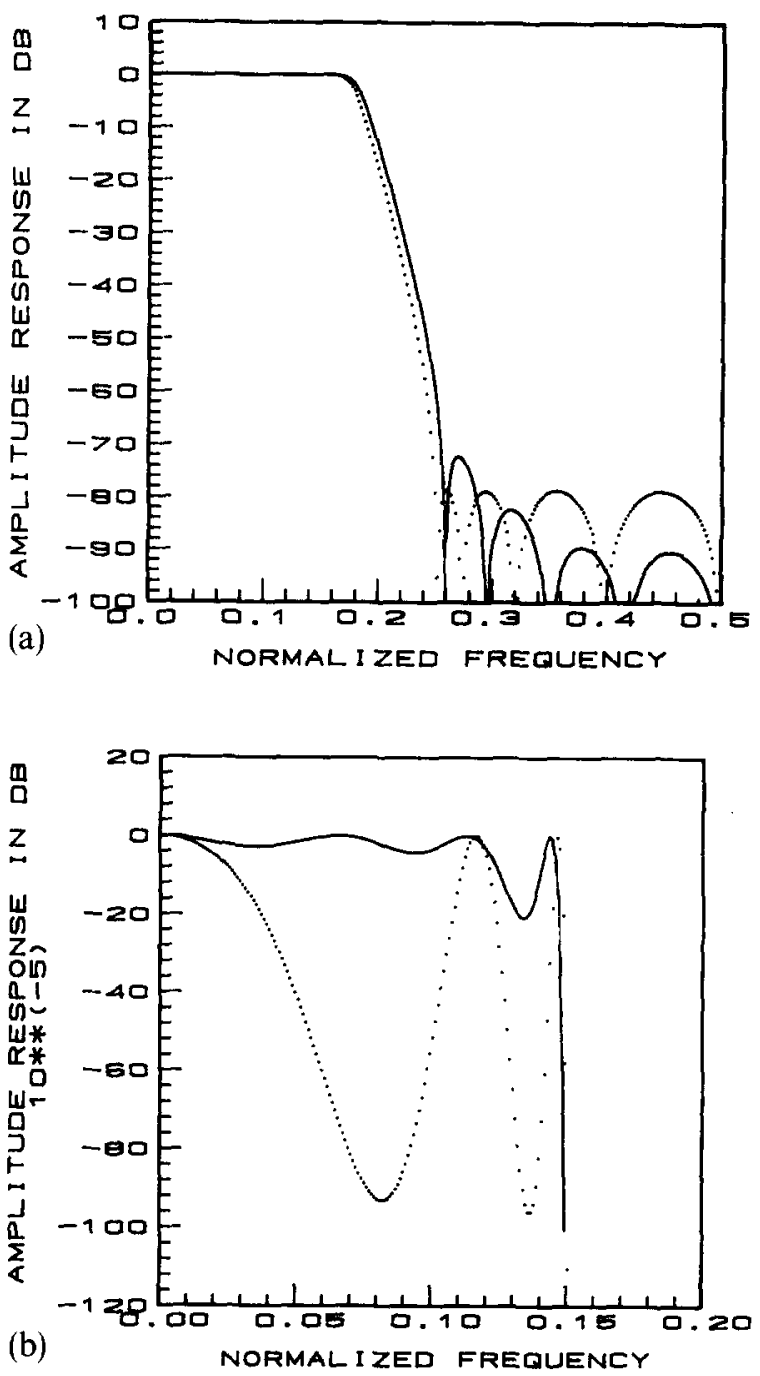

Fig. 1. Example 1: a low-pass filter design, (a) amplitude response, (b) passband detail (multiplied by $10^{-5}$ ). (solid line: paper's method, dotted line: Ansari's method).

and those inside the unit circle are

0.092573 ,

0.152381 ,

$0.328744 \pm \mathrm{j} 0.610513$,

so $N_{1}=4$ and $N_{2}=4$. The response of this filter is shown in Fig. 1(a), while the passband detail is shown in Fig. 1(b) in which the value along the vertical axis is multiplied by $10^{-5}$. Comparing with the results in [3] represented by the dotted line, the 
error for the proposed approach is lower over most of the band, although it has a somewhat larger deviation at the band edges. So the performance of our method is comparable to that in [3].

EXAMPLE 2. It is well known that the interpolation or decimation by a factor of two can be efficiently performed by using symmetric halfband filters. The transfer function of the generalized recursive halfband filter formed by two parallel all-pass sections can be expressed as [1]

$H(z)=\frac{1}{2}\left[H_{1}(z)+H_{2}(z)\right]$,

where

$H_{1}(z)=$

$z^{-1} \frac{a_{1}\left(N_{1}\right)+a_{1}\left(N_{1}-2\right) z^{-2}+\cdots+a_{1}(0) z^{-N_{1}}}{a_{1}(0)+a_{1}(2) z^{-2}+\cdots+a_{1}\left(N_{1}\right) z^{-N_{1}}}$

and

$H_{2}(z)=\frac{a_{2}\left(N_{2}\right)+a_{2}\left(N_{2}-2\right) z^{-2}+\cdots+a_{2}(0) z^{-N_{2}}}{a_{2}(0)+a_{2}(2) z^{-2}+\cdots+a_{2}\left(N_{2}\right) z^{-N_{2}}}$,

in which $N_{1}$ and $N_{2}$ are even integers due to the zero-valued coefficients every two points for halfband filters. Obviously, the design procedures of the halfband filter are similar to those of a general low-pass filter except that the passband cutoff edge $\omega_{\mathrm{p}}$ and the stopband cutoff edge $\omega_{\mathrm{s}}$ are related by

$\omega_{\mathrm{p}}=\pi-\omega_{\mathrm{s}}$.

The corresponding elements in $\boldsymbol{Q}$ for zero-valued coefficients can be deleted which will save design time. In this example, $N=N_{1}+N_{2}=6, \omega_{\mathrm{p}}=$ $0.44 \pi, \omega_{\mathrm{s}}=0.56 \pi$, and

$W(\omega)= \begin{cases}\omega^{2}, & \omega \in \text { passband }, \\ (\pi-\omega)^{2}, & \omega \in \text { stopband }\end{cases}$

are chosen. The obtained poles of $G(z)$ outside the unit circle are

$$
\pm \mathrm{j} 1.479817
$$

and those inside the unit circle are

$$
\begin{aligned}
& \pm \mathrm{j} 0.371431, \\
& \pm \mathrm{j} 0.901054,
\end{aligned}
$$
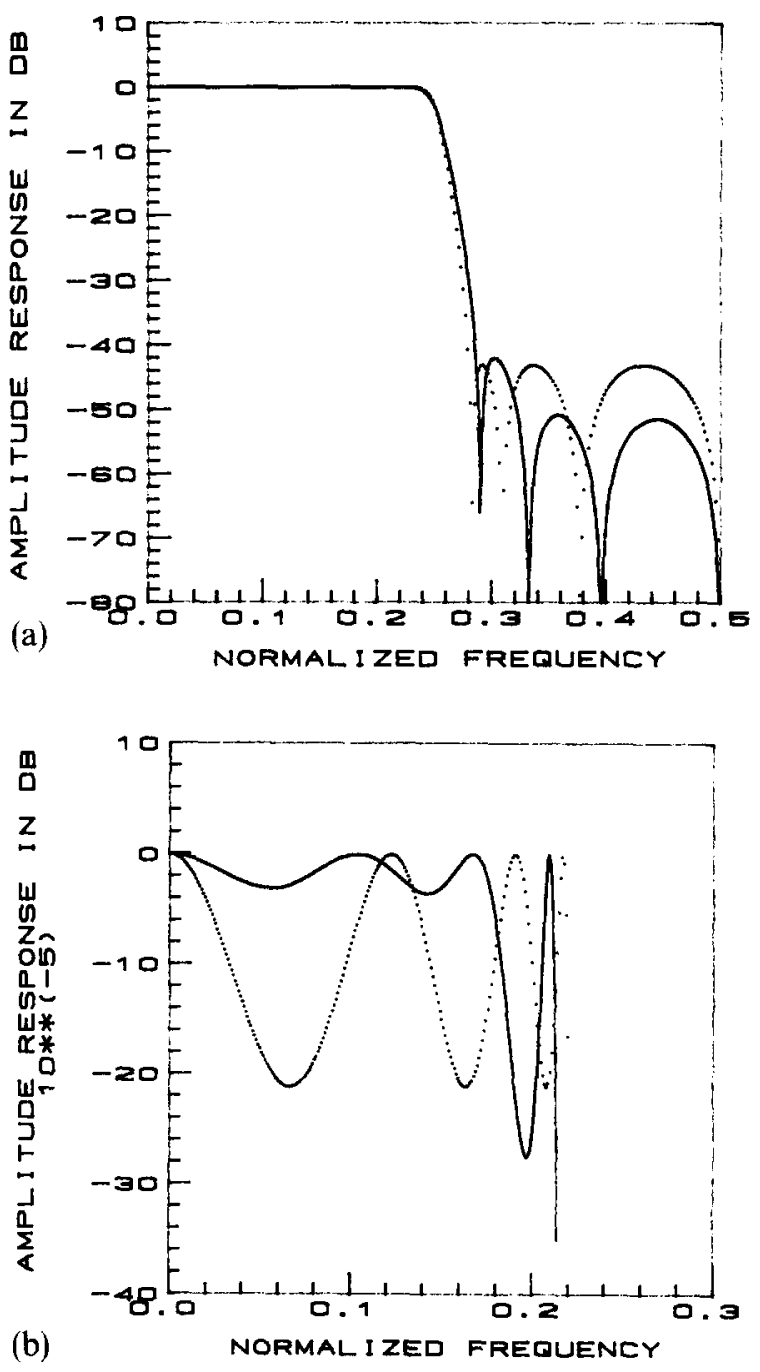

Fig. 2. Example 2: a halfband filter design, (a) amplitude response, (b) passband detail (multiplied by $10^{-5}$ ). (solid line: paper's method, dotted line: Ansari's method).

so $N_{1}=2$ and $N_{2}=4$. The actual response is presented in Fig. 2(a), and Fig. 2(b) shows the passband detail. The overall performance is also comparable to that in [1].

EXAMPLE 3. To show the usefulness of the presented approach, a fourband filter is designed in this example with passbands $[0.18 \pi, 0.38 \pi],[0.76 \pi, \pi]$ and stopbands $[0,0.12 \pi],[0.48 \pi, 0.66 \pi]$ in normalized frequency. $W(\omega)=1$ in passbands and 


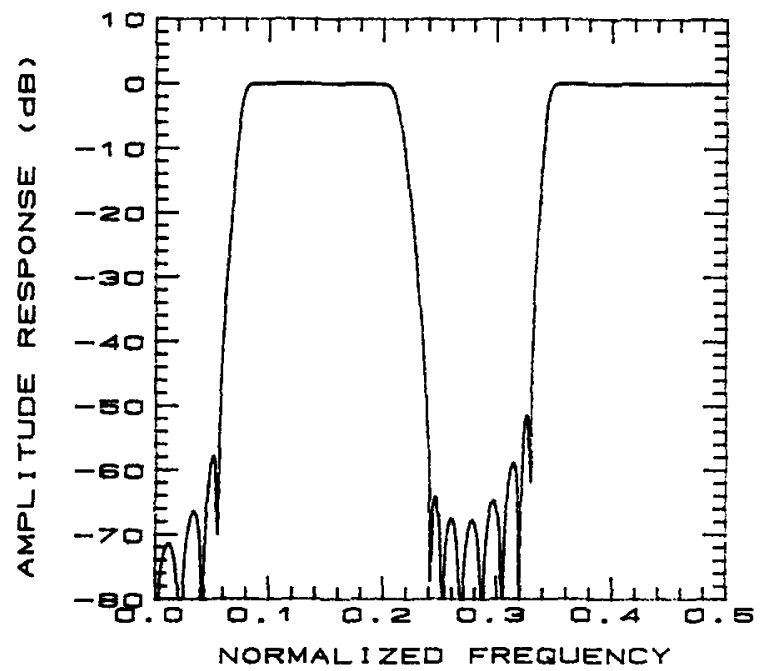

Fig. 3. Example 3: the amplitude response of a four band filter design.

$W(\omega)=5$ in stopbands are used, and $I=1, M=1$, $N_{1}=10$ and $N_{2}=12$ are chosen according to the constraints listed in Table 1 . The obtained amplitude response is shown in Fig. 3.

\section{Design of complex multiband filters using complex IIR all-pass filters}

In general, when the desired response is neither symmetric nor antisymmetric about $\omega=0$, the filter coefficients are complex. In this section, we will be using complex IIR all-pass filters to implement such filters. The overall transfer function is expressed as

$H(z)=\frac{1}{2}\left[H_{1}(z)+H_{2}(z)\right]$,

where

$H_{1}(z)=\frac{\sum_{n=0}^{N_{1}} a_{1}^{*}\left(N_{1}-n\right) z^{-n}}{\sum_{n=0}^{N_{1}} a_{1}(n) z^{-n}}$

and

$H_{2}(z)=\frac{\sum_{n=0}^{N_{2}} a_{2}^{*}\left(N_{2}-n\right) z^{-n}}{\sum_{n=0}^{N_{2}} a_{2}(n) z^{-n}}$,

in which $a_{1}(n)$ and $a_{2}(n)$ are complex-valued filter coefficients and $*$ denotes the complex conjugate operator. It is noted that the factor $(-1)^{1}$ is missing because the frequency responses for complex coefficient filters are generally not symmetric about $\omega=0$. Similarly, Eq. (34) can be rewritten as

$H(z)=\frac{1}{2} H_{1}(z)[1+G(z)]$.

Hence, the design of amplitude response of $H(z)$ can be replaced by the design of the phase response of $G(z)$. This is to say, $G(z)$ and 1 are in-phase in the passband and out-phase in the stopband. Let

$G(z)=\frac{H_{2}(z)}{H_{1}(z)}=\frac{\sum_{n=0}^{N} a(N-n) z^{-n}}{\sum_{n=0}^{N} a(n) z^{-n}}=\frac{z^{-N} F\left(z^{-1}\right)}{F(z)}$,

in which $N=N_{1}+N_{2}$ and

$a(n)=a_{1}^{*}\left(N_{1}-n\right) * a_{2}(n)$,

then

$\arg \left(G\left(\mathrm{e}^{\mathrm{j} \omega}\right)\right)=-N \omega-2 \arg \left(F\left(\mathrm{e}^{\mathrm{j} \omega}\right)\right)$.

So the phase design of $G(z)$ can be converted to the phase design of $F(z)$. If $a_{\mathrm{R}}(n)$ and $a_{\mathrm{I}}(n)$ are the real and imaginary parts of $a(n)$, respectively, then the actual phase of $F(z)$ can be represented by

$$
\begin{aligned}
& \arg \left(F\left(\mathrm{e}^{\mathrm{j} \omega}\right)\right) \\
& \quad=\tan ^{-1} \frac{-\sum_{n=0}^{N} a_{\mathrm{R}}(n) \sin (n \omega)+\sum_{n=0}^{N} a_{\mathrm{I}}(n) \cos (n \omega)}{\sum_{n=0}^{N} a_{\mathrm{R}}(n) \cos (n \omega)+\sum_{n=0}^{N} a_{\mathrm{I}}(n) \sin (n \omega)} \\
& \quad=P_{a}(\omega),
\end{aligned}
$$

which can be rewritten as

$$
\begin{aligned}
& \frac{-\sum_{n=0}^{N} a_{\mathrm{R}}(n) \sin (n \omega)+\sum_{n=0}^{N} a_{\mathrm{I}}(n) \cos (n \omega)}{\sum_{n=0}^{N} a_{\mathrm{R}}(n) \cos (n \omega)+\sum_{n=0}^{N} a_{\mathrm{I}}(n) \sin (n \omega)} \\
& \quad=\tan \left(P_{a}(\omega)\right)=\frac{\sin \left(P_{a}(\omega)\right)}{\cos \left(P_{a}(\omega)\right)} .
\end{aligned}
$$

So

$$
\begin{array}{r}
\sin \left(P_{\mathrm{a}}(\omega)\right)\left[\sum_{n=0}^{N} a_{\mathrm{R}}(n) \cos (n \omega)+\sum_{n=0}^{N} a_{\mathrm{I}}(n) \sin (n \omega)\right] \\
+\cos \left(P_{a}(\omega)\right)\left[\sum_{n=0}^{N} a_{\mathrm{R}}(n) \sin (n \omega)\right. \\
\left.-\sum_{n=0}^{N} a_{1}(n) \cos (n \omega)\right]=0
\end{array}
$$


which can be written in a vector product form

$\boldsymbol{A}^{\mathrm{T}} \boldsymbol{C}(\omega)=0$,

where

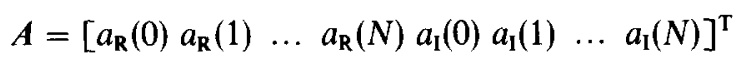

and

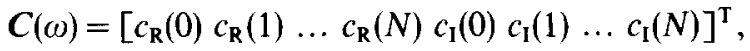

in which

$$
\begin{aligned}
c_{\mathrm{R}}(i) & =\sin \left(P_{a}(\omega)\right) \cos (i \omega)+\cos \left(P_{a}(\omega)\right) \sin (i \omega), \\
0 & \leqslant i \leqslant N
\end{aligned}
$$

and

$c_{\mathrm{I}}(i)=\sin \left(P_{a}(\omega)\right) \sin (i \omega)-\cos \left(P_{a}(\omega)\right) \cos (i \omega)$,

$0 \leqslant i \leqslant N$.

Obviously, the eigenfilter approach can also be used to design such filters, and the error function is

$$
\begin{aligned}
E & =\int_{\boldsymbol{r}} W(\omega)\left[\boldsymbol{A}^{\mathrm{T}} \hat{\boldsymbol{C}}(\omega)\right]^{2} \mathrm{~d} \omega \\
& =\boldsymbol{A}^{\mathrm{T}}\left[\int_{r} W(\omega) \hat{\boldsymbol{C}}(\omega) \hat{\boldsymbol{C}}^{\mathrm{T}}(\omega) \mathrm{d} \omega\right] \boldsymbol{A} \\
& =\boldsymbol{A}^{\mathrm{T}} \boldsymbol{Q} \boldsymbol{A},
\end{aligned}
$$

where

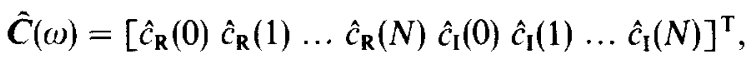

in which

$$
\begin{aligned}
\hat{\mathcal{C}}_{\mathbf{R}}(i) & =\sin \left(P_{\mathrm{F}}(\omega)\right) \cos (i \omega)+\cos \left(P_{\mathrm{F}}(\omega)\right) \sin (i \omega), \\
0 & \leqslant i \leqslant N
\end{aligned}
$$

and

$$
\begin{aligned}
\hat{c}_{1}(i) & =\sin \left(P_{F}(\omega)\right) \sin (i \omega)-\cos \left(P_{F}(\omega)\right) \cos (i \omega), \\
0 & \leqslant i \leqslant N .
\end{aligned}
$$

$P_{F}(\omega)$ is the desired phase response of $F(z)$.

EXAMPLE 4. Consider the desired response of a single-passband filter

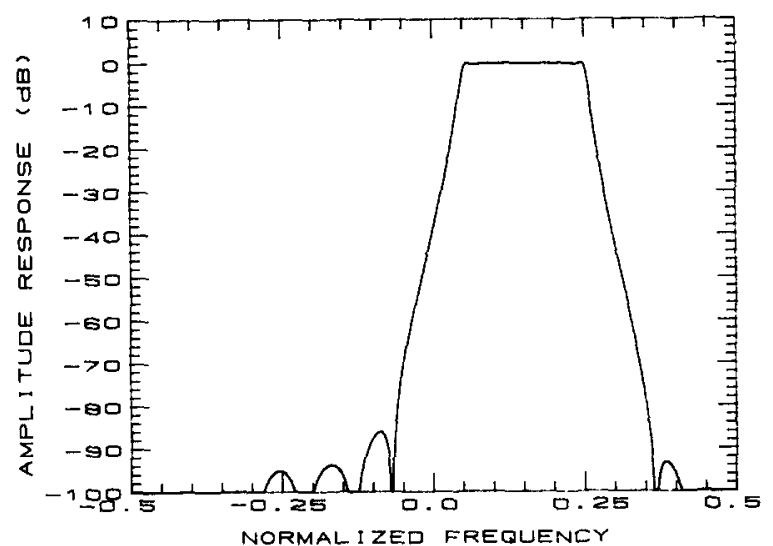

Fig. 4. Example 4: the amplitude response of a singlepassband filter design.

$D(\omega)$

$$
=\left\{\begin{array}{l}
1, \quad 0.1 \pi \leqslant \omega \leqslant 0.5 \pi, \\
0, \quad-\pi \leqslant \omega \leqslant-0.1 \pi \text { and } 0.7 \pi \leqslant \omega \leqslant \pi,
\end{array}\right.
$$

which is approximated by $N_{1}=4$ and $N_{2}=4$ complex all-pass filters. When $W(\omega)=1$ in passband and $W(\omega)=5$ in stopband is used, the poles of $G(z)$ outside the unit circle are

$1.002938+\mathrm{j} 0.3321737$,

$0.1594073+\mathrm{j} 1.162088$,

$-0.0800445-\mathrm{j} 1.646991$,

$0.9206678+\mathrm{j} 0.9400068$,

and the poles inside the unit circle are

$0.3097259+\mathrm{j} 0.704706$,

$-0.02945948-\mathrm{j} 0.6057489$,

$0.7401374+\mathrm{j} 0.3930253$,

$-0.00559021+\mathrm{j} 0.9515541$.

The overall amplitude response is shown in Fig. 4.

EXAMPLE 5. A two passband filter with the desired response 


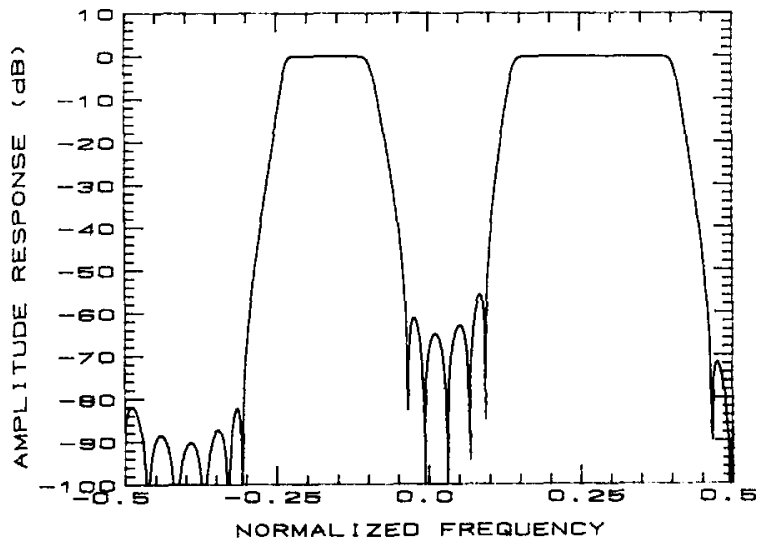

Fig. 5. Example 5: the amplitude response of a two-passband filter design.

$$
\begin{aligned}
& D(\omega)= \\
& \left\{\begin{array}{l}
1, \quad-0.44 \pi \leqslant \omega \leqslant-0.24 \pi \\
\quad \text { and } 0.36 \pi \leqslant \omega \leqslant 0.76 \pi, \\
0, \quad-\pi \leqslant \omega \leqslant-0.6 \pi,-0.08 \pi \leqslant \omega \leqslant 0.2 \pi \\
\quad \text { and } 0.92 \pi \leqslant \omega \leqslant \pi,
\end{array}\right.
\end{aligned}
$$

is designed in this example. The used orders are $N_{1}=5$ and $N_{2}=7$. When $W(\omega)=1$ for all design bands, the obtained poles of $G(z)$ outside the unit circle are

$$
\begin{aligned}
& 1.053653-\mathrm{j} 0.9103779, \\
& 0.8611296+\mathrm{j} 1.072719, \\
& 0.2298666-\mathrm{j} 1.2574408, \\
& -0.8853946+\mathrm{j} 2.802949, \\
& -1.071084+\mathrm{j} 0.8028648,
\end{aligned}
$$

and those inside the unit circle are

$$
\begin{gathered}
0.2472173+j 0.4036998, \\
-0.3905408+j 0.3802253, \\
0.2753842-j 0.5777248, \\
0.7514107-j 0.512103, \\
0.5849311+j 0.7018989, \\
-0.7571492+j 0.5181929, \\
0.0890638-j 0.9249747 .
\end{gathered}
$$

Fig. 5 presents the overall amplitude response.

\section{Conclusions}

In this paper, an efficient method has been proposed to design IIR all-pass filters for synthesizing general multiband filters by minimizing a quadratic measure of the error derived from the phase design of certain filter. The problem of stability is of no concern, and the solution is obtained directly with no need for iteration. Several examples have been presented to demonstrate the efficiency of the present method. Comparing to the existing methods, the performances of our results are comparable to those of [3] and [1], but the proposed approach is more simple and flexible.

\section{References}

[1] R. Ansari, "Elliptic filter design for a class of generalized halfband filters", IEEE Trans. Acoust. Speech Signal Process., Vol. 33, October 1985, pp. 1146-1150.

[2] R. Ansari, "IIR discrete-time Hilbert transformers", IEEE Trans. Acoust. Speech Signal Process., Vol. 35, August 1987, pp. 1116-1119.

[3] R. Ansari and B. Liu, "A class of low-noise computationally efficient recursive digital filters with applications to sampling rate alterations", IEEE Trans. Acoust. Speech Signal Process., Vol. 33, February 1985, pp. 90-97.

[4] A.G. Constantinides and R.A. Valenzuela, 'An efficient and modular transmultiplexer design", IEEE Trans. Comm., Vol. 30, July 1982, pp. 1629-1641.

[5] B. Nobel and J.W. Daniel, Applied Linear Algebra, Prentice-Hall, Englewood Cliffs, NJ, 1977.

[6] S.C. Pei and J.J. Shyu, "Design of FIR Hilbert transformers and differentiators by eigenfilters", IEEE Trans. Circuits and Systems, Vol. 35, November 1988, pp. 1457-1461.

[7] S.C. Pei and J.J. Shyu, "Eigenfilter design of higher order digital differentiators", IEEE Trans. Acoust. Speech Signal Process., Vol. 37, April 1989, pp. 505-511.

[8] L.R. Rabiner and B. Gold, Theory and Application of Digital Signal Processing, Prentice-Hall, Englewood Cliffs, NJ, 1975.

[9] P.P. Vaidyanathan and T.Q. Nguyen, "Eigenfilter: A new approach to least-squares FIR filter design and applications including Nyquist filters", IEEE Trans. Circuits and Systems, Vol. 34, January 1987, p. 11-23.

[10] P.P. Vaidyanathan, S.K. Mitra and Y. Neuvo, "A new approach to the realization of low-sensitivity IIR digital filters", IEEE Trans. Acoust. Speech Signal Process., Vol. 34, April 1986, pp. 350-361.

[11] P.P. Vaidyanathan, P.A. Regalia and S.K. Mitra, "Design of doubly complementary IIR digital filters using a single complex allpass filters, with multirate applications", IEEE Trans. Circuits and Systems, Vol. 34, April 1987, pp. 378-389. 